\title{
Ductal Carcinoma In Situ of the Breast: Perspectives on Tumor Subtype and Treatment
}

\author{
Yufei Liu $\mathbb{D},{ }^{1,2}$ Kangquan Shou $\mathbb{D}^{3},{ }^{3}$ Juanjuan Li, ${ }^{4}$ Qi Wu, ${ }^{4}$ Yuchang Hu, ${ }^{1,2}$ Junjie Wang, ${ }^{5}$ \\ Chunyu Cao, ${ }^{6}$ and Qing Wang ${ }^{1,2}$ \\ ${ }^{1}$ Institute of Pathology, China Three Gorges University, Yichang 443000, China \\ ${ }^{2}$ Department of Pathology, Yichang Central People's Hospital, Yichang 443003, China \\ ${ }^{3}$ Department of Orthopaedics, Yichang Central People's Hospital, Yichang 443003, China \\ ${ }^{4}$ Department of Breast and Thyroid Surgery, Renming Hospital of Wuhan University, Wuhan 430060, China \\ ${ }^{5}$ Department of Obstetrics and Gynecology, Ren He Hospital of China Three Gorges University, Yichang 443001, China \\ ${ }^{6}$ Hubei Key Laboratory of Tumor Microenvironment and immunotherapy, China Three Gorges University, Yichang 443000, China
}

Correspondence should be addressed to Yufei Liu; 402762573@qq.com

Received 5 October 2019; Accepted 18 April 2020; Published 28 May 2020

Academic Editor: John P. Geisler

Copyright (C) 2020 Yufei Liu et al. This is an open access article distributed under the Creative Commons Attribution License, which permits unrestricted use, distribution, and reproduction in any medium, provided the original work is properly cited.

\begin{abstract}
Objective. To evaluate ductal carcinoma in situ (DCIS) characteristics and the effect of different treatment strategies. Patients and Methods. Using data with known hormone receptor (HoR) and human epidermal growth factor receptor 2 (HER2) status obtained by the Surveillance, Epidemiology, and End Results (SEER) program from 2010-2014, the study was conducted to investigate tumor subtype-specific differences in various characteristics, overall survival (OS), and breast cancer-specific mortality (BCSM). Results. A total of 3415 patients with DCIS were eligible. Compared with HoR+/HER- subgroup, patients with triple-negative (TN) and HoR/HER+ were commonly higher in grade, larger in size, and tended to receive mastectomy $(P<0.05)$. The multivariate analysis revealed that patients with TN were more likely to have a poorer OS and show a higher breast cancer-specific mortality compared with the HoR+/HER- subgroup $(P<0.05)$. Multivariate analysis on the history of local treatment and surgery showed patients receiving breast-conserving surgery $(\mathrm{BCS})$ plus radiotherapy $(\mathrm{R})$ and BCS plus axillary lymph node dissection was likely to improve OS without affecting breast cancer-specific mortality $(P<0.05)$. Conclusion. The results demonstrate that DCIS associated with TN subtype portends poor prognosis. Meanwhile, BCS plus R was a preferable option and resulted in survival rates better than those achieved with mastectomy, and SLNB should be considered as an appropriate assessment of axillary staging in patients with DCIS.
\end{abstract}

\section{Introduction}

Ductal carcinoma in situ (DCIS) of the breast describes lesions comprised of abnormal epithelial cells that are completely confined within breast ducts without invasion beyond the basement membrane. In the majority of patients, DCIS is primarily viewed as the indicator of invasive breast cancer (IBC) $[1,2]$. Notably, the positive detection rate of DCIS increased rapidly after the introduction of mammography as a population screening tool and substantially increased at a slower rate [3-5].

As previously mentioned, DCIS has been characterized as a type of breast cancer with small in size, low grade, and estrogen receptor (ER) and progesterone receptor (PR) positive expression, but HER2 testing is not a routine part of the pathologic evaluation $[6,7]$. However, studies suggested that DCIS lesions with high nuclear grade are often ER negative and HER2 overexpression. Furthermore, HER2 overexpression has also been demonstrated to be associated with the reduction of survival, and targeting HER2 has been a potential treatment strategy for HER2-overexpressing DCIS.

The traditional treatment of DCIS is mastectomy, whereas breast conservation surgery (BCS) is a feasible surgical option for selected patients. In addition to BCS, outcomes related to axillary lymph node dissection in DCIS have not been validated in well-controlled clinical trials. Based on 
the current guideline, SLNB has been recommended as a less invasive method to stage patients with early invasive ductal carcinoma than axillary lymph node dissection (ALND), but routine use of SLNB for DCIS is not supported. We speculate that SLNB would be approved as an effective method in detecting the status of axillary lymph nodes (ALNs). The benefit of radiotherapy, in terms of a significantly reduced risk of local recurrence in those undergoing BCS, has been demonstrated by several large randomized controlled trials $[8,9]$. However, it is not clear whether radiotherapy should be appropriate in selected low-risk cases. The clinical characteristics of DCIS and optimal treatment strategy are topics of uncertainty and concern for both patients and clinicians. Therefore, this article aims at evaluating DCIS characteristics and the effect of different treatment strategies, with special emphasis on the role of subtypes of DCIS, BCS, and SLNB.

\section{Patients and Methods}

2.1. Patient Selection. All the patient data were from the National Cancer Institute's Surveillance, Epidemiology, and End Results (SEER) program between 2010 and 2014. 2010 was selected as the starting point of our measurements as SEER began HER2 status collection in 2010. We had used the International Classification of Diseases for Oncology, 3rd edition (ICD-O-3) histopathology codes to extract all cases with DCIS (codes 8500,8523 ), basing on a scheme used previously [10]. The selected cases were all with known breast subtypes. Cases that did not receive surgery, for which the histologic types were unknown, or were diagnosed at autopsy were excluded. Demographic variables included age at diagnosis $(<35,35-49,50-64$, and $>65$ years). Cancer characteristics were classified by grade (well, moderately, poorly, undifferentiated, and unknown), tumor size $(\leq 10,10-20$, 20-50, and $>50 \mathrm{~mm}$ ), and laterality (right, left, others, and unknown). Treatment characteristics included receipt of radiation therapy (no, yes, and unknown). All the subtypes were characterized according to the breast subtype variable as HoR+/HER2-, HoR+/HER2+, HoR-/HER2+, and triplenegative (TN). Patients were categorized as receiving BCS (surgery of primary site variable values of 20-24) and mastectomy (surgery of primary site variable values of 30-80). Because the type of axillary surgery was not reported within SEER, replacement of 1-5 lymph nodes removed was regarded as axillary lymph node dissection groups and $>5$ lymph nodes removed was taken for axillary lymph node dissection (ALND) groups, as shown in previous studies [11]. We characterized several subgroups named "local treatment" (mastectomy (M), BCS only, and BCS with radiotherapy (BCS $+\mathrm{R})$ and "Surgery" (M+ALND, M+SLNB, BCS+ALND, BCS+SLNB) (Table 1). Specifically, the radiotherapy is the adjuvant therapy only after breast-conserving surgery, not for the patients who received mastectomy without axillary lymph node dissection. And the radiotherapy is limited to the whole breast not including the axillary area.

The two primary outcomes in our study were OS and breast cancer-specific mortality. Vitality status was recorded as "alive" or "dead" in the SEER dataset. Survival time (in months) was calculated for each patient using the "Com- pleted Months of Follow-up" in the SEER database. OS was determined by patients who were alive at the end of the study period or who were alive at their last follow-up. Breast cancer-specific mortality was determined by patients whose cause of death was due to breast cancer with patients who were alive at the end of the study period, had died due to other causes, or who were alive at their last follow-up. Cases without survival times were classified as unknown and removed from this study.

2.2. Statistical Analysis. Patient demographics and cancerrelated characteristics were compared within subgroups using Chi-square or Fisher's exact tests. Survival outcomes on OS and breast cancer-specific mortality were estimated using the weighted Kaplan-Meier method, and variables were compared using the log-rank test in the subgroups. Univariate and multivariate Cox proportional hazard regressions were used to obtain hazard ratios (HRs) and their respective 95\% confidence intervals and show the strength of the estimated relative risk; these approaches were applied to model the relationship between potential covariates and either OS or breast cancer-specific mortality. All statistical analyses and all charts of survival probabilities were performed using SPSS 19.0 (IBM Corporation, Armonk, NY). A two-sided P value $<0.05$ was considered statistically significant.

\section{Results}

A total of 3415 patients with DCIS were eligible during the study period from 2010 to 2014. A total of 2050 DCIS cases within HoR+/HER2- subgroup, 765 in HoR+/HER2+, 365 in HoR-/HER2+, and 235 TN (triple negative) patients had available information and were included within this study.

Differences in patient demographics, cancer characteristics, treatments, and outcomes within subgroups were summarized in Table 2. Compared with HoR+/HER2- subgroup, patients with TN were commonly higher in grade, larger in size, and older in age (each $P<0.05$ ). Patients within HoR-/ HER2+ subgroup were more universally higher in grade and larger in size compared with HoR+/HER2- subgroup. For treatment options, patients with TN and HoR-/HER2+ subgroup tended to receive mastectomy, and all cases were increasingly treated by SLNB.

Weighted Kaplan-Meier analysis was used to determine OS and breast cancer-specific mortality in the subgroups based on breast subtype. Survival curves for the subgroups were generated (Figures 1(a) and 2(a)). At the median follow up 42 months, patients with TN had an OS of $97.0 \%$ compared with $98.6 \%$ in the HoR+/HER2- subgroup $(P<0.05)$. Further, the breast cancer-specific mortality rate was $1.5 \%$ for the TN group compared with $0.2 \%$ for the HoR+/HER2- subgroup $(P<0.05)$. We used multivariate analysis based on the weighted Kaplan-Meier results. All the prognostic factors that predicted OS and breast cancer-specific mortality were analyzed on multivariate analysis (Table 2). In the multivariate analysis, patients with TN were more likely to have a poorer OS and a higher breast cancer-specific mortality were showed compared with the HoR+/HER2- subgroup (OS, 
TABle 1: Patient characteristics within subgroups.

\begin{tabular}{|c|c|c|c|c|c|}
\hline Variables & HoR+/HER- $N=2050(\%)$ & $\mathrm{HoR}+/ \mathrm{HER}+N=765(\%)$ & HoR-/HER+ $N=365(\%)$ & TN $N=235(\%)$ & $P$ value* \\
\hline Follow-up (months) & $28.47 \pm 17.29$ & $28.12 \pm 17.72$ & $27.50 \pm 17.03$ & $30.48 \pm 17.45$ & \\
\hline Age at diagnosis $(\mathrm{y})$ & & & & & $P<0.001$ \\
\hline$<35$ & $11(0.5)$ & $9(1.2)$ & $3(0.8)$ & $0(0.0)$ & \\
\hline $35-49$ & $417(20.3)$ & $173(22.6)$ & $68(18.6)$ & $36(15.3)$ & \\
\hline $50-64$ & $808(39.4)$ & $346(45.2)$ & $171(46.8)$ & $108(46.0)$ & \\
\hline$\geq 65$ & $814(39.7)$ & $237(31.0)$ & $123(33.7)$ & $91(38.7)$ & \\
\hline Grade & & & & & $P<0.001$ \\
\hline Well & $363(17.7)$ & $39(5.1)$ & $5(1.4)$ & $7(3.0)$ & \\
\hline Moderately & $810(39.5)$ & $193(25.2)$ & $38(10.4)$ & $45(19.1)$ & \\
\hline Poorly & $553(27.0)$ & $406(53.1)$ & $258(70.7)$ & $143(60.9)$ & \\
\hline Undifferentiated & $55(2.7)$ & $35(4.6)$ & $21(5.8)$ & $12(5.1)$ & \\
\hline Unknown & $269(13.1)$ & $92(12.0)$ & $43(11.8)$ & $28(11.9)$ & \\
\hline Tumor size $(\mathrm{mm})$ & & & & & $P<0.001$ \\
\hline$\leq 10$ & $847(41.3)$ & $278(36.3)$ & $109(29.9)$ & $73(31.1)$ & \\
\hline $10-20$ & $396(19.3)$ & $179(23.4)$ & $89(24.4)$ & $69(29.4)$ & \\
\hline $20-50$ & $209(10.2)$ & $103(13.5)$ & $64(17.5)$ & $28(11.9)$ & \\
\hline$>50$ & $60(2.9)$ & $29(3.8)$ & $17(4.7)$ & $7(3.0)$ & \\
\hline Unknown & $538(26.2)$ & $176(23.0)$ & $86(23.6)$ & $58(24.7)$ & \\
\hline Laterality & & & & & 0.254 \\
\hline Left & $1051(51.3)$ & $388(50.7)$ & $201(55.1)$ & $110(46.8)$ & \\
\hline Right & $999(48.7)$ & $377(49.3)$ & $164(44.9)$ & $125(53.2)$ & \\
\hline Radiotherapy & & & & & 0.480 \\
\hline None/unknown & $1052(51.3)$ & $380(49.7)$ & $183(50.1)$ & $130(55.3)$ & \\
\hline Yes & $998(48.7)$ & $385(50.3)$ & $182(49.9)$ & $105(44.7)$ & \\
\hline Local treatment & & & & & 0.009 \\
\hline M & $574(28.0)$ & $252(32.9)$ & $126(34.5)$ & $90(38.3)$ & \\
\hline BCS & $1402(68.4)$ & $489(63.9)$ & $222(60.8)$ & $140(59.6)$ & \\
\hline None & $69(3.4)$ & $21(2.7)$ & $15(4.1)$ & $4(1.7)$ & \\
\hline Unknown & $5(0.2)$ & $3(0.4)$ & $2(0.5)$ & $1(0.4)$ & \\
\hline Axillary treatment & & & & & $P<0.001$ \\
\hline None & $1285(62.7)$ & $432(56.5)$ & $188(51.5)$ & $121(51.5)$ & \\
\hline SLNB & $663(32.3)$ & $290(37.9)$ & $152(41.6)$ & $101(43.0)$ & \\
\hline ALND & $84(4.1)$ & $37(4.8)$ & $23(6.3)$ & $12(5.1)$ & \\
\hline Unknown & $18(0.9)$ & $6(0.8)$ & $2(0.5)$ & $1(0.4)$ & \\
\hline Status & & & & & $P<0.001$ \\
\hline Alive & $1998(97.5)$ & $745(97.4)$ & $356(97.5)$ & $218(92.8)$ & \\
\hline Dead & $52(2.5)$ & $20(2.6)$ & $9(2.5)$ & $17(7.2)$ & \\
\hline Breast cancer & $5(0.2)$ & $3(0.4)$ & $2(0.5)$ & $6(2.6)$ & \\
\hline Other & $47(2.3)$ & $17(2.2)$ & $7(1.9)$ & $11(4.7)$ & \\
\hline
\end{tabular}

${ }^{*} P$ values calculated by Pearson Chi -squared testing; bold if statistically significant, $P<0.05$. y: years; mm: millimeter; y: years; BCS: breast-conserving surgery; HoR: hormone receptor; TN: triple negative; M: mastectomy; SLNB: sentinel lymph node biopsy; ALND: axillary lymph node dissection.

$P=0.01$, adjusted hazard ratio $(\mathrm{aHR})=3.092$; breast cancerspecific mortality, $P=0.012$, aHR $=7.725)$.

During the follow-up period, we analyzed adjuvant radiotherapy, showing that patients undergoing radiotherapy showed a higher OS without affecting breast cancer-specific mortality (Figures 1 (b) and 2(b), OS, $P=0.004$, aHR $=0.314$; breast cancer-specific mortality, $P=0.35$, aHR $=0.514)$.
Furthermore, our findings demonstrated that compared with the patients treated with $M+A L N D$, those patients underwent with BCS+SLNB were likely to display a better OS (Figures 1 (c) and 2(c), $P=0.014$, aHR $=0.298$ ). Additionally, multivariate analysis of OS on history of local treatment showed patients receiving BCS combined with radiotherapy $(\mathrm{R})$ was likely to improve OS compared with 
TABLE 2: Cox proportional hazards regression model analysis of overall survival (OS) and breast cancer-specific mortality (BCSM).

\begin{tabular}{|c|c|c|c|c|}
\hline \multirow{2}{*}{ Variables } & \multicolumn{2}{|c|}{ OS } & \multicolumn{2}{|c|}{ BCSM } \\
\hline & aHR (95\% CI) & $P$ value & aHR (95\% CI) & $P$ value \\
\hline \multicolumn{5}{|l|}{ Age at diagnosis (y) } \\
\hline$<35$ & Reference & & Reference & \\
\hline $35-49$ & $564.1(0.0,1.49 \mathrm{E} 58)$ & 0.922 & $5.092(0.0,1.58 \mathrm{E} 177)$ & 0.994 \\
\hline $50-64$ & $550.8(0.0,1.45 \mathrm{E} 58)$ & 0.923 & $1591.7(0.0,3.37 \mathrm{E} 179)$ & 0.972 \\
\hline$\geq 65$ & $3134.5(0.0,8.24 \mathrm{E} 58)$ & 0.902 & $8686.1(0.0,1.84 \mathrm{E} 180)$ & 0.965 \\
\hline \multicolumn{5}{|l|}{ Grade } \\
\hline Well & Reference & & Reference & \\
\hline Moderately & $4.965(0.647,38.096)$ & 0.123 & $2275.2(0.0,3.711 \mathrm{E} 22)$ & 0.732 \\
\hline Poorly & $1.318(0.158,11.018)$ & 0.799 & $3735.7(0.0,6.985 \mathrm{E} 22)$ & 0.716 \\
\hline Undifferentiated & $2.882(0.243,34.136)$ & 0.401 & $69.918(0.0,1.380 \mathrm{E} 30$ & 0.898 \\
\hline \multicolumn{5}{|l|}{ Laterality } \\
\hline Left & Reference & & Reference & \\
\hline Right & $1.913(0.983,3.724)$ & 0.056 & $14.934(0.685,325.57)$ & 0.086 \\
\hline \multicolumn{5}{|l|}{ Tumor size (mm) } \\
\hline$\leq 10$ & Reference & & Reference & \\
\hline $10-20$ & $1.411(0.602,3.307)$ & 0.428 & $0.003(0.0,2405831.4)$ & 0.579 \\
\hline $20-50$ & $0.633(0.172,2.338)$ & 0.493 & $0.138(0.003,7.196)$ & 0.327 \\
\hline$>50$ & $1.750(0.445,6.888)$ & 0.423 & $0.267(0.009,7.787)$ & 0.443 \\
\hline \multicolumn{5}{|l|}{ Subtype } \\
\hline HoR+/HER- & Reference & & Reference & \\
\hline $\mathrm{HoR}+/ \mathrm{HER}+$ & $1.269(0.480,3.354)$ & 0.630 & $1.595(0.083,13.188)$ & 30.732 \\
\hline HoR-/HER+ & $2.518(0.2918,6.909)$ & 0.073 & $0.365(0.007,17.853)$ & 0.611 \\
\hline $\mathrm{TN}$ & $3.878(1.619,9.289)$ & 0.002 & $17.093(0.467,625.28)$ & 0.122 \\
\hline \multicolumn{5}{|l|}{ Radiotherapy } \\
\hline No & Reference & & Reference & \\
\hline Yes & $0.261(0.083,0.891)$ & 0.021 & $3.751(0.110,127.66)$ & 0.463 \\
\hline \multicolumn{5}{|l|}{ Local treatment } \\
\hline M & Reference & & Reference & \\
\hline BCS & $1.274(0.512,3.171)$ & 0602 & $0.0(0.0,4.739 \mathrm{E} 42)$ & 0.886 \\
\hline $\mathrm{BCS}+\mathrm{R}$ & $0.270(0.101,0.718)$ & 0.009 & $0.001(0.0,1.492 \mathrm{E} 18)$ & 0.764 \\
\hline \multicolumn{5}{|l|}{ Surgery } \\
\hline M+ALND & Reference & & Reference & \\
\hline $\mathrm{M}+\mathrm{SLNB}$ & $0.5484(0.236,1.275)$ & 0.163 & $0.014(0.001,0.389)$ & 0.012 \\
\hline BCS+ALND & $0.891(0.170,4.666)$ & 0.891 & $0.0(0.0)$ & 0.984 \\
\hline BCS+SLNB & $0.265(0.099,0.705)$ & 0.008 & $0.0(0.0,2.337 \mathrm{E} 178)$ & 0.945 \\
\hline
\end{tabular}

${ }^{*} P$ values calculated by Log-rank testing; bold if statistically significant, $P<0.05$. M: mastectomy; BCS: breast-conserving surgery; R: radiotherapy; HoR: hormone receptor; TN: triple negative; LN: lymph node; SLNB: sentinel lymph node biopsy; ALND: axillary lymph node dissection. aHR: adjusted hazard ratio (adjusted for age at diagnosis, race, grade, tumor size, laterality, ER, PR, HER2, subtype, radiotherapy, local treatment, and surgery).

those in the mastectomy subgroup $(P=0.016$, aHR $=$ $0.374)$, but did not affect breast cancer-specific mortality (Figures 1(d) and 2(d)).

\section{Discussion}

In our previous work [11], we found that a poorer survival in TN subtype when adjusting for other factors based on a large population-based cohort of cases diagnosed with breast carcinoma in situ (BCIS), furthermore, in the current study, with a longer follow-up duration (median follow-up, 42 months), we found similar results in a large series of DCIS histological subtypes. Furthermore, we focus on DCIS. In our series, there was a dramatic difference within the subgroups of local treatment, radiotherapy, and surgical strategy only for OS.

In previous studies, a new Van Nuys Prognostic Index (VNPI) is used as the independent predictor of local recurrence, which derives a new formula, including tumor size, margin width, pathologic grade, and age [12]. However, our research showed that tumor size, nuclear grade, and age were not the prognostic factors for OS or breast cancer-specific 


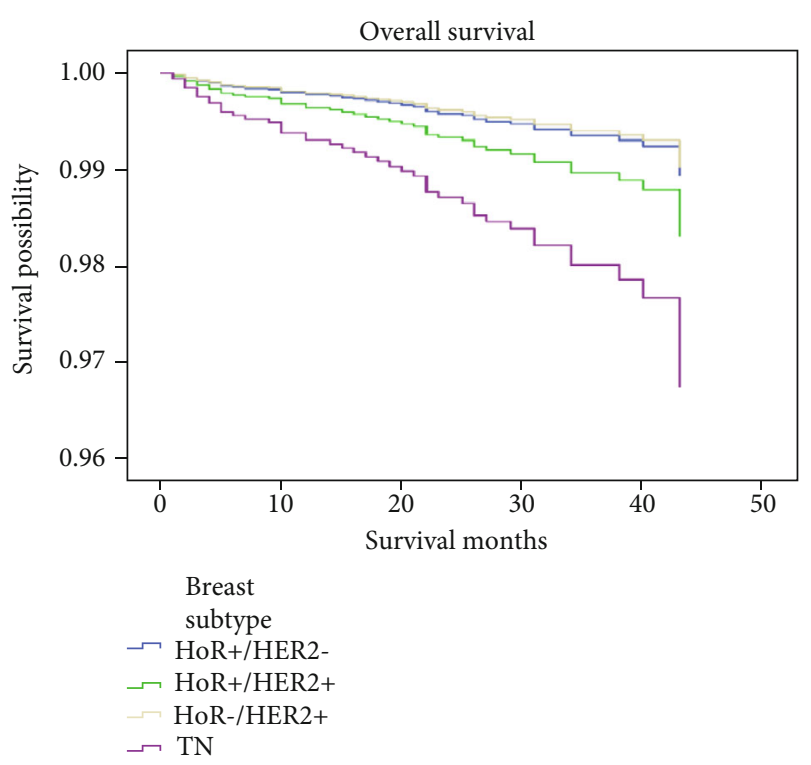

(a)

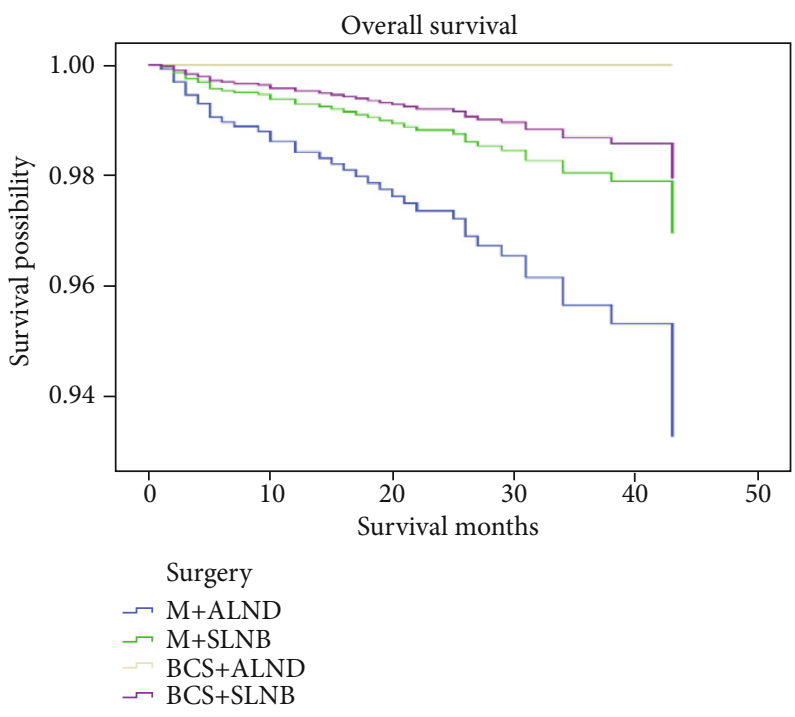

(c)



(b)



(d)

FIgURE 1: Weighted Kaplan-Meier curves of overall survival (OS) in subgroup analysis. (a) OS is based on breast subtype. (b) OS is based on radiotherapy. (c) OS is based on surgery. (d) OS is based on local treatment.

mortality. In the current study, patients within the HoR/HER2+ subgroup were higher in grade and larger in size compared with HoR+/HER2- subgroup. The correlation between HER2 and tumor behavior has been described previously. That study revealed that HER2 was overexpressed in 24/31 (77\%) patients who experienced local relapse for DCIS [13]. Whereas, another study showed that HER2 overexpression may not be the key factor in the progression of DCIS transforming to invasive carcinoma, and HER2 gene amplification is inversely related to the invasive progression in DCIS patients $[14,15]$. Simultaneously, the precise incidence of HER2 overexpression in many cases of DCIS is unclear. A retrospective analysis showed that HER2 was overexpressed in $61 \%$ of cases with DCIS [16]. In contrast, Roses et al. [17] reported 106 patients with DCIS noted
HER 2 overexpression in only $37 \%$ of cases. Furthermore, they also described an association between HER2 overexpression and the detection of invasive foci on surgical specimens. However, we analyzed that HER2 status was not correlated with survival adjusting for other prognostic factors. This conclusion remains to be further confirmed by prospective trial.

The TN subtype of DCIS was diagnosed seldom, possibly because the tumor progression of TN subtype is quicker than other types of breast cancer. In consistent with the previous study, our study reported that the TN subtype may be much faster in progression than other breast cancer types [15]. In this study, data showed that patients with TN subtype tended to be higher in grade, larger in size, and older in age, and the prognosis of TN subtype were more likely to be poor. Some 


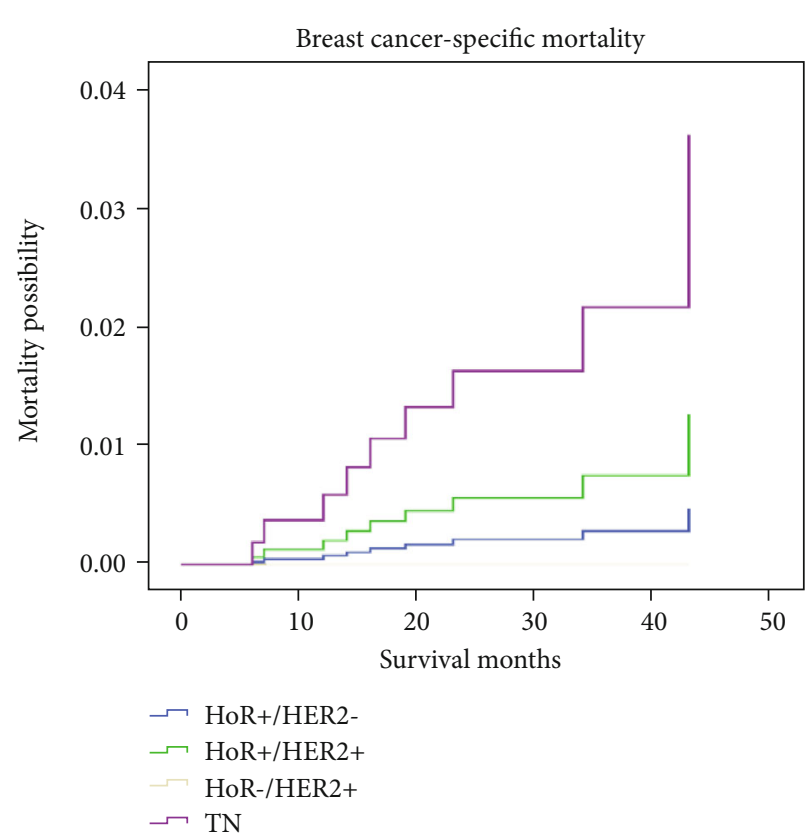

(a)

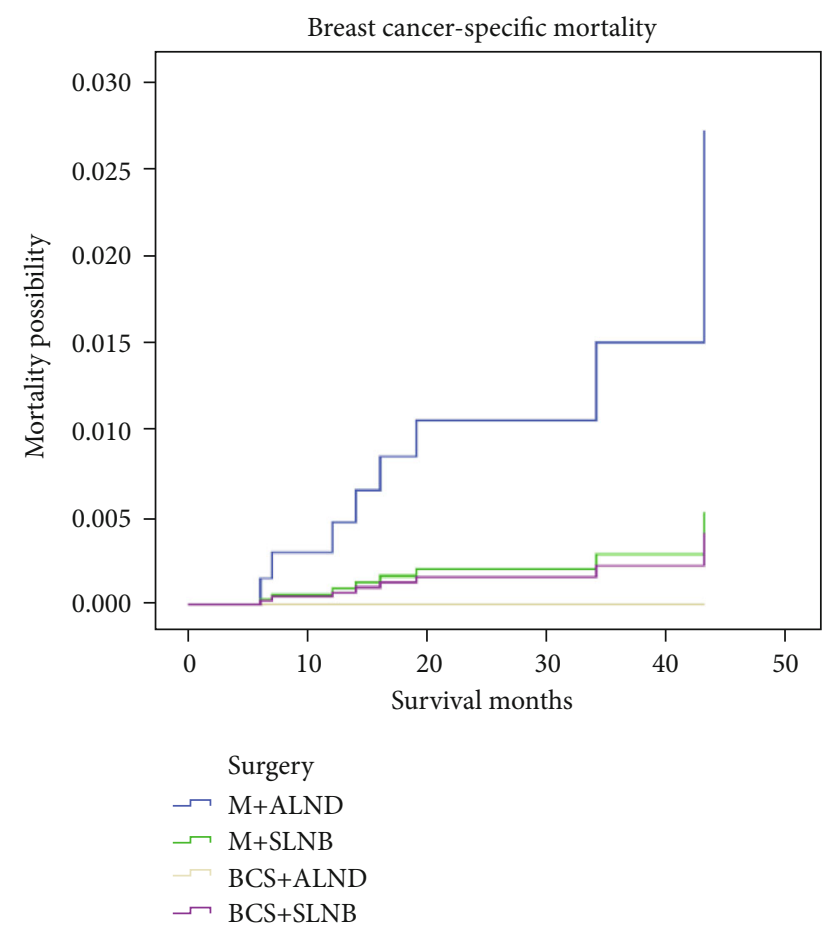

(c)



(b)



$$
\begin{aligned}
& \begin{array}{l}
\text { Local } \\
\text { treatment }
\end{array} \\
- & \text { M } \\
- & \text { BCS } \\
- & \text { BCSR }
\end{aligned}
$$

(d)

Figure 2: Weighted Kaplan-Meier curves of breast cancer-specific mortality (BCSM) in subgroup analysis. (a) BCSM is based on breast subtype. (b) BCSM is based on radiotherapy. (c) BCSM is based on surgery. (d) BCSM is based on local treatment.

reports had suggested that TN subtype DCIS may be a potential precursor to $\mathrm{TN}$ invasive cancer $[18,19]$, and a more frequent and rapid progression from DCIS to invasive cancer was related to the comedo subtype of DCIS $[20,21]$. Consequently, some unrecognized mechanisms or features helping TN subtype in progression should be further studied.
Currently, the treatment strategy of DCIS was controversial. Although mastectomy was curative for approximately all of patients with DCIS $[22,23]$, mastectomy is still considered as overtreatment. Although radiotherapy has been proved to decrease local recurrence compared with BCS alone [24], it may also lead to overtreatment for the low-risk patients. Local excision only was regarded as an appropriate surgery 
for patients with low-risk DCIS in several studies [12, 25]. Furthermore, several retrospective studies [26-30] reported that BCS resulted in consistently higher rates of local recurrence (range, $8 \%-34 \%$ ) compared with patients treated by $\mathrm{BCS}+\mathrm{R}$ (range, 0\%-17\%). Similarly, our results also presented that patients receiving $\mathrm{BCS}+\mathrm{R}$ was likely to improve OS compared with those in the mastectomy subgroup. Therefore, BCS combined with radiotherapy was a preferable alternative for patients with DCIS. According to the previous study, axillary metastases were found in approximately 1-2\% of DCIS cases [31], therefore, the need for axillary staging still remains necessary $[32,33]$. Previously, SLNB was considered feasible for patients with DCIS [34-36]. In our study, patients undergoing BCS+SLNB showed better survival than those receiving $M+A L N D$, which can be attributed to the radiotherapy. However, routine use of SLNB is only recommended for high-risk DCIS [35].

About the molecular profiles of DCIS, some studies had confirmed that DCIS was high expression of estrogen receptor (ER) associated with low-grade lesions, but positive for cerbB-2 (HER2), Ki67, and p53 associated with high-grade lesions [31, 37, 38]. A recent case-control study suggested that DCIS cases with triple positive for p16, COX-2, and Ki67 had a significantly higher rate of progression to invasive breast cancer than those of negative for these biomarkers (8year risks of subsequent invasive cancer $19.6 \%$ and $4.1 \%$, respectively) [39]. Simultaneously, several studies confirmed that HoR negativity, high S-phase fraction, abnormal DNA ploidy, P53 overexpression, and HER2 over-expression were associated with more aggressive tumor behavior in DCIS [40-43]. Gene expression profiling was likely to enhance our understanding on DCIS behavior and its relation to invasive breast cancer. The findings of several studies $[40,44]$ had recorded differential expression patterns and identified new facets of the earliest stage of breast cancer progression. More molecular and genetic studies that predict local recurrence and progression to invasive breast cancer independent of standard prognostic markers would be required, and the difference in survival must continue to be monitored.

The main limitations of our study were heterogeneous population and retrospective setting. Furthermore, the information on systemic therapy and margin control was insufficient. In doing so, HER2 targeted therapy and novel adjuvant hormone therapy should remain utilized for the management of DCIS to improve survival. Additionally, we had no specific information on the type of axillary operation, and thus used the number of lymph nodes removed as a surrogate of the type of axillary surgery (SLNB vs. ALND).

\section{Conclusions}

Despite the limitations listed above, our study demonstrated that DCIS appears to alter prognosis associated with TN subtype. Meanwhile, BCS plus R was a preferable option and resulted in survival rates better than those achieved with mastectomy, and SLNB should be considered as an appropriate assessment of axillary staging in patients with DCIS. However, the surgical treatment plan must be chosen for the strength of helpful clinical and imaging assessment. Further researches are needed to minimize variation in modes of treatment and establish a standardized management approach.

\section{Abbreviation}

DCIS: Ductal carcinoma in situ

IBC: Invasive breast cancer

ER: Estrogen receptor

PR: Progesterone receptor

BCS: Breast conservation surgery

ALND: Axillary lymph node dissection

SEER: Surveillance, epidemiology, and end results

HRs: Hazard ratios.

\section{Data Availability}

This is an open access article distributed under the Creative Commons Attribution License, which permits unrestricted use, distribution, and reproduction in any medium, provided the original work is properly cited.

\section{Ethical Approval}

This article does not contain any studies with human participants or animals materials performed by any of the authors.

\section{Conflicts of Interest}

All authors declare that they have no conflict of interest.

\section{Authors' Contributions}

Yufei Liu and Kangquan Shou contributed equally to this work.

\section{Acknowledgments}

This work was partially supported by Natural Science Foundation of Hubei Province to Prof. Chunyu Cao (Grant NO: 2017CFB556), and this work was also supported by an Open Foundation for Tumor Microenvironment and Immunotherapy Key Laboratory of Hubei Province in China grant to Dr. Yufei Liu (Grant NO: 2018KZL03).

\section{References}

[1] M. J. Van de Vijver and H. Peterse, "The diagnosis and management of pre-invasive breast disease: pathological diagnosis-problems with existing classifications," Breast Cancer Research, vol. 5, no. 5, 2003.

[2] Q. Sun, M. Dong, Z. Wang et al., "Selenium-enriched polysaccharides from Pyracantha fortuneana (Se-PFPs) inhibit the growth and invasive potential of ovarian cancer cells through inhibiting $\beta$-catenin signaling," Oncotarget, vol. 7 , no. 19, pp. 28369-28383, 2016.

[3] B. A. Virnig, T. M. Tuttle, T. Shamliyan, and R. L. Kane, "Ductal carcinoma in situ of the breast: a systematic review of incidence, treatment, and outcomes," Journal of the National Cancer Institute, vol. 102, no. 3, pp. 170-178, 2010. 
[4] S. A. Joslyn, "Ductal carcinoma in situ: trends in geographic, temporal, and demographic patterns of care and survival," The Breast Journal, vol. 12, no. 1, pp. 20-27, 2006.

[5] K. Kerlikowske, "Epidemiology of ductal carcinoma in situ," Journal of the National Cancer Institute Monographs, vol. 2010, no. 41, pp. 139-141, 2010.

[6] E. M. Ward, C. E. DeSantis, C. C. Lin et al., "Cancer statistics: breast cancer in situ," CA: a cancer journal for clinicians, vol. 65, no. 6, pp. 481-495, 2015.

[7] W. Zhou, K. Jirström, R. M. Amini et al., "Molecular subtypes in ductal carcinoma in situ of the breast and their relation to prognosis: a population-based cohort study," BMC Cancer, vol. 13, no. 1, 2013.

[8] N. Vapiwala, E. Harris, W. T. Hwang, and L. J. Solin, "Longterm outcome for mammographically detected ductal carcinoma in situ managed with breast conservation treatment: prognostic significance of reexcision," Cancer Journal, vol. 12, no. 1, pp. 25-32, 2006.

[9] B. Cutuli, C. Cohen-Solal-le Nir, B. de Lafontan et al., "Breastconserving therapy for ductal carcinoma in situ of the breast: the French Cancer Centers' experience," International journal of radiation oncology, biology, physics, vol. 53, no. 4, pp. 868879, 2002.

[10] C. I. Li, K. E. Malone, B. S. Saltzman, and J. R. Daling, "Risk of invasive breast carcinoma among women diagnosed with ductal carcinoma in situ and lobular carcinoma in situ, 19882001," Cancer, vol. 106, no. 10, pp. 2104-2112, 2006.

[11] J. Wang, E. A. Mittendorf, A. A. Sahin et al., "Outcomes of sentinel lymph node dissection alone vs. axillary lymph node dissection in early stage invasive lobular carcinoma: a retrospective study of the surveillance, epidemiology and end results (SEER) database," PLoS One, vol. 9, no. 2, e89778, 2014.

[12] M. J. Silverstein, "The University of Southern California/Van Nuys prognostic index for ductal carcinoma in situ of the breast," American journal of surgery, vol. 186, no. 4, pp. 337343, 2003.

[13] N. Bijker, J. L. Peterse, L. Duchateau et al., "Histological type and marker expression of the primary tumour compared with its local recurrence after breast-conserving therapy for ductal carcinoma in situ," British journal of cancer, vol. 84, no. 4, pp. 539-544, 2001.

[14] D. C. Allred, G. M. Clark, R. Molina et al., "Overexpression of HER-2/neu and its relationship with other prognostic factors change during the progression of in situ to invasive breast cancer," Human pathology, vol. 23, no. 9, pp. 974979, 1992.

[15] S. Kurbel, "In search of triple-negative DCIS: tumor-type dependent model of breast cancer progression from DCIS to the invasive cancer," Tumour biology : the journal of the International Society for Oncodevelopmental Biology and Medicine, vol. 34, no. 1, pp. 1-7, 2013.

[16] Y. Horimoto, E. Tokuda, A. Arakawa, T. Kosaka, M. Saito, and F. Kasumi, "Significance of HER2 protein examination in ductal carcinoma in situ," The Journal of surgical research, vol. 167, no. 2, pp. e205-e210, 2011.

[17] R. E. Roses, E. C. Paulson, A. Sharma et al., "HER-2/neu overexpression as a predictor for the transition from in situ to invasive breast cancer," Cancer epidemiology, biomarkers \& prevention: a publication of the American Association for Cancer Research, cosponsored by the American Society of Preventive Oncology, vol. 18, no. 5, pp. 1386-1389, 2009.
[18] B. B. Bryan, S. J. Schnitt, and L. C. Collins, "Ductal carcinoma in situ with basal-like phenotype: a possible precursor to invasive basal-like breast cancer," Modern Pathology, vol. 19, no. 5, pp. 617-621, 2006.

[19] S. E. Clark, J. Warwick, R. Carpenter, R. L. Bowen, S. W. Duffy, and J. L. Jones, "Molecular subtyping of DCIS: heterogeneity of breast cancer reflected in pre-invasive disease," British journal of cancer, vol. 104, no. 1, pp. 120-127, 2011.

[20] S. E. Pinder and I. O. Ellis, "The diagnosis and management of pre-invasive breast disease: ductal carcinoma in situ (DCIS) and atypical ductal hyperplasia $(\mathrm{ADH})$-current definitions and classification," Breast Cancer Research, vol. 5, no. 5, pp. 254-257, 2003.

[21] J. F. Simpson, "Update on atypical epithelial hyperplasia and ductal carcinoma in situ," Pathology, vol. 41, no. 1, pp. 3639, 2009.

[22] C. Shah, J. Wobb, B. Manyam et al., "Management of ductal carcinoma in situ of the breast: a review," JAMA Oncology, vol. 2, no. 8, pp. 1083-1088, 2016.

[23] A. Lebeau and T. Kuhn, "Updates in the treatment of ductal carcinoma in situ of the breast," Current Opinion in Obstetrics \& Gynecology, vol. 28, no. 1, pp. 49-58, 2016.

[24] J. P. Julien, N. Bijker, I. S. Fentiman et al., "Radiotherapy in breast-conserving treatment for ductal carcinoma in situ: first results of the EORTC randomised phase III trial 10853," Lancet, vol. 355, no. 9203, pp. 528-533, 2000.

[25] M. J. Silverstein, D. N. Poller, J. R. Waisman et al., "Prognostic classification of breast ductal carcinoma-in-situ," Lancet, vol. 345, no. 8958, pp. 1154-1157, 1995.

[26] E. Y. Weng, G. J. F. Juillard, R. G. Parker, H. R. Chang, and J. A. Gornbein, "Outcomes and factors impacting local recurrence of ductal carcinoma in situ," Cancer, vol. 88, no. 7, pp. $1643-$ 1649, 2000.

[27] M. K. Jha, V. S. Avlonitis, C. D. M. Griffith et al., “Aggressive local treatment for screen-Ėdetected DCIS results in very low rates of recurrence," European Journal of Surgical Oncology, vol. 27, no. 5, pp. 454-458, 2001.

[28] I. de Mascarel, F. Bonichon, G. MacGrogan et al., “Application of the van nuys prognostic index in a retrospective series of 367 ductal carcinomas in situ of the breast examined by serial macroscopic sectioning: practical considerations," Breast Cancer Research and Treatment, vol. 61, no. 2, pp. 151-159, 2000.

[29] A. Ringberg, L. Anagnostaki, H. Anderson, I. Idvall, M. Fernö, and South Sweden Breast Cancer Group, "Cell biological factors in ductal carcinoma in situ (DCIS) of the breast- relationship to ipsilateral local recurrence and histopathological characteristics," European Journal of Cancer, vol. 37, no. 12, pp. 1514-1522, 2001.

[30] A. G. Douglas-Jones, J. Logan, J. M. Morgan, R. Johnson, and R. Williams, "Effect of margins of excision on recurrence after local excision of ductal carcinoma in situ of the breast," Journal of Clinical Pathology, vol. 55, no. 8, pp. 581-586, 2002.

[31] G. D. Leonard and S. M. Swain, "Ductal carcinoma in situ, complexities and challenges," Journal of the National Cancer Institute, vol. 96, no. 12, pp. 906-920, 2004.

[32] A. Katz, I. Gage, S. Evans et al., "Sentinel lymph node positivity of patients with ductal carcinoma in situ or microinvasive breast cancer," American Journal of Surgery, vol. 191, no. 6, pp. 761-766, 2006.

[33] R. Sakr, C. Bezu, I. Raoust et al., "The sentinel lymph node procedure for patients with preoperative diagnosis of ductal 
carcinoma in situ: risk factors for unsuspected invasive disease and for metastatic sentinel lymph nodes," International Journal of Clinical Practice, vol. 62, no. 11, pp. 1730-1735, 2008.

[34] R. F. D. van la Parra, M. F. Ernst, P. C. Barneveld, J. M. Broekman, M. J. C. M. Rutten, and K. Bosscha, "The value of sentinel lymph node biopsy in ductal carcinoma in situ (DCIS) and DCIS with microinvasion of the breast," European Journal of Surgical Oncology, vol. 34, no. 6, pp. 631-635, 2008.

[35] H. M. Shapiro-Wright and T. B. Julian, "Sentinel lymph node biopsy and management of the axilla in ductal carcinoma in situ," Journal of the National Cancer Institute Monographs, vol. 2010, no. 41, pp. 145-149, 2010.

[36] R. Sakr, E. Barranger, M. Antoine, H. Prugnolle, E. Darai, and S. Uzan, "Ductal carcinoma in situ: value of sentinel lymph node biopsy," Journal of Surgical Oncology, vol. 94, no. 5, pp. 426-430, 2006.

[37] W. F. Anderson and K. C. Chu, "Ductal carcinoma in situ of the breast," The New England Journal of Medicine, vol. 351, pp. 399-402, 2004.

[38] H. M. Kuerer, C. T. Albarracin, W. T. Yang et al., "Ductal carcinoma in situ: state of the science and roadmap to advance the field," Journal of Clinical Oncology, vol. 27, no. 2, pp. 279-288, 2009.

[39] K. Kerlikowske, A. M. Molinaro, M. L. Gauthier et al., "Biomarker expression and risk of subsequent tumors after initial ductal carcinoma in situ diagnosis," Journal of the National Cancer Institute, vol. 102, no. 9, pp. 627-637, 2010.

[40] M. Aubele, A. Mattis, H. Zitzelsberger et al., "Extensive ductal carcinoma in situ with small foci of invasive ductal carcinoma: evidence of genetic resemblance by CGH," International Journal of Cancer, vol. 85, no. 1, pp. 82-86, 2000.

[41] T. Baqai and S. Shousha, "Oestrogen receptor negativity as a marker for high-grade ductal carcinoma in situ of the breast," Histopathology, vol. 42, no. 5, pp. 440-447, 2003.

[42] H. Buerger, F. Otterbach, R. Simon et al., "Comparative genomic hybridization of ductal carcinomain situ of the breast?evidence of multiple genetic pathways," The Journal of Pathology, vol. 187, no. 4, pp. 396-402, 1999.

[43] E. Provenzano, J. L. Hopper, G. G. Giles, G. Marr, D. J. Venter, and J. E. Armes, "Biological markers that predict clinical recurrence in ductal carcinoma in situ of the breast," European Journal of Cancer, vol. 39, no. 5, pp. 622-630, 2003.

[44] C. B. Moelans, R. A. de Weger, H. N. Monsuur, A. H. J. Maes, and P. J. van Diest, "Molecular differences between ductal carcinoma in situ and adjacent invasive breast carcinoma: a multiplex ligation-dependent probe amplification study," Analytical Cellular Pathology (Amsterdam), vol. 33, no. 3-4, pp. 165-173, 2010. 